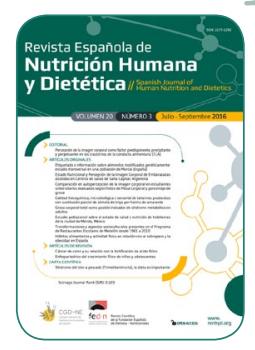

ORIGINAL

\title{
Calidad fisicoquímica, microbiológica y sensorial de tallarines producidos con sustitución parcial de sémola de trigo por harina de amaranto
}

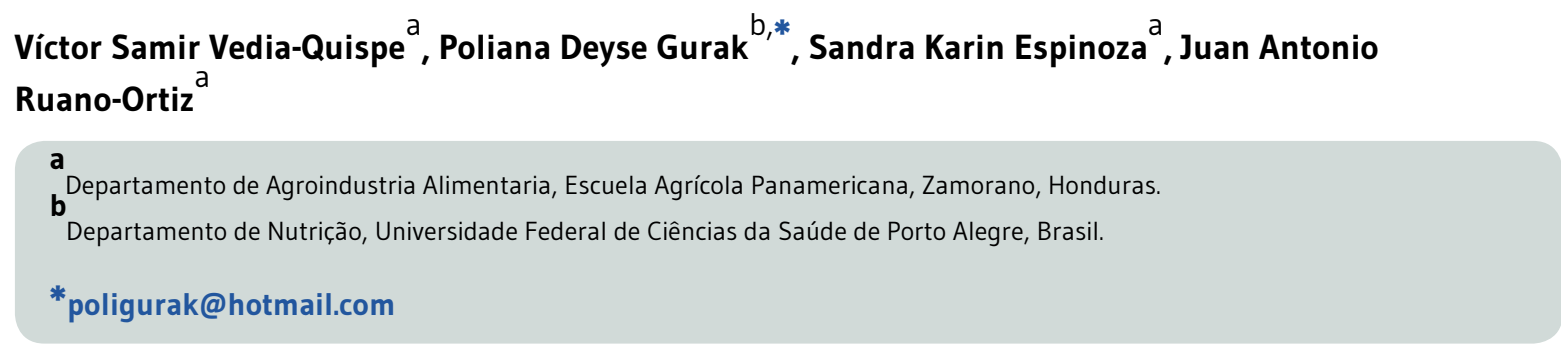

Recibido el 24 de febrero de 2016; aceptado el 12 de agosto de 2016.

\section{PALABRAS CLAVE}

Análisis de alimentos;

Harina;

Amaranthus;

Fibra alimentaria;

Minerales;

Sabor.
Calidad fisicoquímica, microbiológica y sensorial de tallarines producidos con sustitución parcial de sémola de trigo por harina de amaranto

\section{RESUMEN}

Introducción: La pasta es un alimento de consumo masivo y de alta aceptabilidad a nivel mundial, debido a su bajo costo, su facilidad de preparación y almacenamiento. Generalmente es elaborada con sémola de trigo y agua. El proceso de sustituir la sémola de trigo por harina de amaranto promueve el desarrollo de nuevos productos y mejora el perfil nutricional de la pasta. El objetivo del presente estudio fue evaluar las propiedades fisicoquímicas, microbiológicas y sensoriales de tallarines con sustitución parcial de sémola de trigo por harina integral de amaranto y harina de amaranto crudo.

Material y Métodos: Se evaluó el efecto de la sustitución parcial (20\% y $30 \%$ ) de sémola de trigo por harina de amaranto crudo y harina de amaranto integral en las características fisicoquímicas, microbiológicas, de calidad y de parámetros de aceptación sensorial de tallarines.

Resultados: El mejor tratamiento fue la combinación de $70 \%$ sémola de trigo y $30 \%$ harina de amaranto crudo, donde el sabor fue el factor que influyó en la aceptación general y algunos parámetros de calidad presentaron correlación con las respuestas sensoriales. Todas las pastas presentaron calidad sanitaria e inocuidad alimentaria.

Conclusiones: La sustitución parcial de harina de amaranto sea cruda o integral incrementó en un $60 \%$ el contenido de fibra y en más de $140 \%$ el calcio y hierro en los tallarines elaborados. 


\section{KEYWORDS}

Food Analysis;

Flour;

Amaranthus;

Dietary Fiber;

Minerals;

Taste.

Physicochemical, microbiological and sensory quality of noodles produced with partial replacement of wheat semolina by amaranth flour

\section{ABSTRACT}

Introduction: Pasta is a worldwide high consumption and acceptability food due to its low cost, easy preparation and storage. Pasta is usually made of edible wheat semolina and water. The replacement of wheat semolina by amaranth flour stimulates the development of new products and improves nutritional profile of pasta. The aim of this study was to assess physicochemical, microbiological and sensory properties of noodles made with partially replaced wheat semolina by whole grain and raw amaranth flours.

Material and Methods: We evaluated the effect of the partial substitution (20\% and $30 \%$ ) of wheat semolina using raw amaranth and whole grain amaranth flours in physicochemical, microbiological, quality characteristics and sensory analysis of acceptance.

Results: The best treatment was the combination of $70 \%$ wheat semolina and $30 \%$ raw amaranth, where flavor was the factor in the overall acceptance, and some quality parameters correlated with the sensory responses. All pastas show sanitary quality and food safety.

Conclusions: The partial substitution of amaranth flour, either raw or whole grain, improved significantly physicochemical characteristics of fiber with an increase of $60 \%$ and $140 \%$ in minerals (calcium and iron) in the noodles.

\section{CITA}

Vedia-Quispe VS, Gurak PD, Espinoza SK, Ruano-Ortiz JA. Calidad fisicoquímica, microbiológica y sensorial de tallarines producidos con sustitución parcial de sémola de trigo por harina de amaranto. Rev Esp Nutr Hum Diet. 2016; 20(3): 190- 197. doi: 10.14306/renhyd.20.3.215

\section{InTRoducción}

El desarrollo de alimentos de consumo masivo y que aporten mayor calidad nutricional, además de mejorar la salud y el bienestar del consumidor es necesario. Entre ellos, la pasta es un alimento de gran consumo y de alta aceptabilidad a nivel mundial debido a su bajo costo, su facilidad de preparación y almacenamiento ${ }^{1}$. Hoy en día la pasta es parte de la canasta familiar en Latinoamérica².

La elaboración de pastas enriquecidas se logra agregando materias primas que ayuden a mejorar el perfil nutricional en las pastas ${ }^{3}$. Dentro de los pseudocereales que pueden ser utilizados, el amaranto (Amaranthus caudatus), que es originario de los Andes y se mantuvo como elemento básico desde el tiempo prehispánico ${ }^{4}$, es una opción.

El amaranto contiene alto valor proteico, fibras y riqueza en minerales (Fe, Ca y $\mathrm{Zn}$ ); estos atributos lo convierten en una buena alternativa como ingrediente para pastas y contribuyen a promover la variedad culinaria y valoración de los gra- nos étnicos de Latinoamérica ${ }^{5-7}$. Además, el reemplazo del trigo por amaranto en un 20 a $25 \%$ tiene un aporte de 14 a $17 \mathrm{~g}$ de proteína en $100 \mathrm{~g}$ de pasta ${ }^{8}$.

En la presente investigación se evaluaron propiedades fisicoquímicas, microbiológicas y sensoriales de tallarines con sustitución parcial ( $20 \%$ y $30 \%$ ) de sémola de trigo por harina integral de amaranto y harina de amaranto crudo.

\section{$\longrightarrow$ \\ MATERIAL Y MÉTODOS}

Se trabajó con $10 \mathrm{~kg}$ de sémola de trigo (ST), $5 \mathrm{~kg}$ de harina integral de amaranto (HIA: Amaranthus hypochondriacus, producto tostado, con eliminación parcial de pericarpio, tamizado y molido) y $5 \mathrm{~kg}$ de harina de amaranto crudo (HAC: Amaranthus hypochondriacus, producto integral secado y molido) para todo el estudio, considerando cada tratamiento y cada repetición por separado. Iniciando el proceso, las harinas pasaron por un tamiz estándar $N^{\circ} 40$ de 8 pulgadas de diámetro obteniendo una harina de $425 \mu \mathrm{m}$. 
Se realizó un diseño de bloques completos al azar con un arreglo factorial de $2 \times 2$, siendo los bloques cada repetición y los factores fueron los tipos de harina (HIA y HAC) compuestas por cinco formulaciones con tres repeticiones (con $250 \mathrm{~g}$ ) por tratamiento preparadas con goma guar (1\%), aceite (2\%) y agua destilada (33\%) y diferentes proporciones de las harinas: i) pasta enriquecida con HIA en concentración 20\% para 80\% de ST, (80ST:20HIA); ii) pasta enriquecida con HIA en concentración $30 \%$ para $70 \%$ de ST (70ST:30HIA); iii) pasta enriquecida con HAC con $20 \%$ para $80 \%$ de ST (80ST:20HAC); iv) pasta enriquecida con HAC con $30 \%$ para $70 \%$ de ST (70ST:30HAC); v) pasta con $100 \%$ de ST (control). Estas proporciones fueron escogidas tras pruebas preliminares con concentraciones de 10, 20, 30, 40 y 50\% de HAC o HAl.

Los ingredientes secos fueron homogenizados en la mezcladora por $4 \mathrm{~min}$ a $60 \mathrm{rpm}$ con el objetivo de evitar la absorción de agua de forma no uniforme y que esto provocara que la pasta se quebrase. Se agregó agua destilada a 65 ${ }^{\circ} \mathrm{C}$ mezclada con goma guar, para evitar la encapsulación de las proteínas y minerales por los fitatos del amaranto. El mezclado duró $10 \mathrm{~min}$ a $180 \mathrm{rpm}$. De este proceso se obtuvo una masa elástica sin pegajosidad lista para el amasado. La masa fue laminada 5 veces para moldearla bien y 4 veces para reducir el grosor de la pasta y obtener tallarines estándar con un ancho de $6 \mathrm{~mm}$ y un grosor de $1 \mathrm{~mm}$. Todo lo anterior con el propósito de estandarizar la masa, provocar que la red de gluten retenga los almidones y evitar que se deshaga la pasta al momento de la cocción ${ }^{9,10}$. Después la pasta fue deshidratada a $45^{\circ} \mathrm{C}$ por $12 \mathrm{~h}$ y luego secada al ambiente por $6 \mathrm{~h}\left(23^{\circ} \mathrm{C} \pm 3\right)$, y finalmente, los tallarines fueron envasados en bolsas de polietileno transparente planas, calibre 175 con cierre hermético.

Los análisis fisicoquímicos realizados fueron humedad (AOAC 952.08); cenizas (11AOAC 923.03); grasa (AOAC 2003.06); proteína cruda (AOAC 2001.11); fibra cruda (AOAC 962.09); hierro y calcio (AOAC 985.35). El extracto libre de nitrógeno se obtuvo por la diferencia entre el peso de la muestra y la suma de los porcentajes de grasa, fibra cruda, proteína cruda y ceniza. Este cálculo permite obtener la cantidad de carbohidratos de cada producto ${ }^{11}$. Para el análisis de actividad de agua se utilizó equipo AQUA $L A B^{\circledR}$ modelo 3 TE. La muestra utilizada en cada unidad experimental por triplicado fue de $5.5 \pm 0.5 \mathrm{~g}$. A temperatura promedio de $24^{\circ} \mathrm{C}$ con un tiempo promedio de lectura por muestra de $3 \mathrm{~min}$.

Los análisis microbiológicos realizados fueron mesófilos aerobios y coliformes totales ${ }^{12}$. Los análisis de calidad ejecutados: cocción ${ }^{13}$ con los parámetros evaluados de aumento de peso, aumento de volumen y tiempo de cocción; gelatinización, color (Colorflex ${ }^{\circledR}$, modelo 45/0 HunterLab
Reston), acidez alcohólica ${ }^{14}$, textura con medición de la fuerza en Newtons y la ruptura en milímetros y tensión (Brookfield CT3) ${ }^{15}$.

Posteriormente se realizó el análisis sensorial afectivo de aceptación (escala hedónica de 9 puntos), donde el nivel de aceptabilidad se da dentro del rango 1 "me disgusta muchísimo" y 9 "me gusta muchísimo". Este ensayo contó con un panel de 25 personas no entrenadas seleccionadas para cada repetición (3 veces). Los panelistas no recibieron ningún tipo de entrenamiento para desarrollar la prueba. Los tratamientos fueron evaluados después del proceso de cocción sirviendo a cada panelista (sin aderezos y acompañamiento de otro tipo de alimentos). Los atributos evaluados fueron: apariencia, olor, color, sabor, textura y aceptación general.

Se ejecutó un análisis de varianza (ANDEVA) para evaluar la significancia del modelo y de esta manera determinar si existen diferencias estadísticas significativas entre las características analizadas de los 5 tratamientos. También se utilizó separación de medias LsMeans para determinar cuál es la mejor combinación que satisface a los panelistas.

\section{Resultados}

En la Tabla 1 se observa la composición química de las materias primas utilizadas y formulaciones de tallarines. En las formulaciones de tallarines, se puede inferir que el contenido de fibra saludable incrementó en más de 4,8 veces cuando fue comparado con el control con la adición de amaranto a la formulación. No hubo diferencia significativa entre 80ST:20HAC y 70ST:30HIA. El incremento de grasa fue significativo en comparación de los tallarines control, mientras que los tallarines con amaranto 70ST:30HIA y 70ST:30HAC no presentaron diferencias significativas, aunque tuvieron un incremento de 2,8 veces y 2,9 veces, respectivamente, más grasa cuando fue comparado con el control. Mientras los tallarines 80ST:20HAC y 80ST:20HIA obtuvieron un aumento de 1,9 veces de grasa, los cuales no tuvieron diferencias significativas entre ellos. El contenido de proteína también incrementó significativamente. El control tuvo 13,18\% de proteína, el tratamiento $80 \mathrm{ST}: 20 \mathrm{HIA}$ no fue significativo, mientras que los tallarines de 70ST:30HAC tuvieron el mayor incremento de proteína (14,90\%), cuando fue comparado con la pasta control.

El tratamiento con mayor contenido de extracto libre de nitrógeno fue el control con $74,73 \%$. El que tuvo menor valor fue el tratamiento 70ST:30HAC con $69,03 \%$, seguido por $80 \mathrm{ST}: 20 \mathrm{HIA}$ con $70,53 \%$. Mientras que no hubo diferencia significativa entre los tratamientos 70ST:30HIA y 80ST:20HAC. 
Tabla 1. Composición de las materias primas y composición de las cinco formulaciones de tallarines. Datos en base seca $(\mathrm{g} / 100 \mathrm{~g})$.

\begin{tabular}{l|c|c|c|c|c|c}
\multicolumn{1}{c}{ Muestra } & Humedad & Ceniza & Fibra & Grasa & Proteína & ELN \\
\hline Materias primas & & & & & & \\
\hline ST & $11,47 \pm 0,02^{\mathrm{a}}$ & $2,41 \pm 0,21^{\mathrm{a}}$ & $0,12 \pm 0,01^{\mathrm{c}}$ & $0,47 \pm 0,11^{\mathrm{b}}$ & $13,34 \pm 0,09^{\mathrm{c}}$ & $72,19 \pm 0,71^{\mathrm{c}}$ \\
HAC & $2,95 \pm 0,11^{\mathrm{b}}$ & $2,89 \pm 0,04^{\mathrm{a}}$ & $6,01 \pm 0,03^{\mathrm{a}}$ & $7,57 \pm 0,23^{\mathrm{a}}$ & $19,02 \pm 0,28^{\mathrm{a}}$ & $61,56 \pm 0,63^{\mathrm{a}}$ \\
HIA & $8,47 \pm 0,05^{\mathrm{c}}$ & $2,17 \pm 0,05^{\mathrm{b}}$ & $4,31 \pm 0,21^{\mathrm{b}}$ & $6,73 \pm 0,28^{\mathrm{a}}$ & $15,78 \pm 0,46^{\mathrm{b}}$ & $62,53 \pm 0,21^{\mathrm{b}}$ \\
\hline Formulaciones de tallarines & & & & & & \\
\hline Control (ST) & $7,80 \pm 0,83^{\mathrm{b}}$ & $1,06 \pm 0,17^{\mathrm{c}}$ & $0,16 \pm 0,02^{\mathrm{d}}$ & $1,08 \pm 0,17^{\mathrm{c}}$ & $13,18 \pm 0,14^{\mathrm{d}}$ & $74,73 \pm 0,84^{\mathrm{a}}$ \\
70ST:3OHAC & $7,74 \pm 0,39^{\mathrm{b}}$ & $1,66 \pm 0,08^{\mathrm{a}}$ & $1,48 \pm 0,26^{\mathrm{a}}$ & $3,20 \pm 0,42^{\mathrm{a}}$ & $14,90 \pm 0,46^{\mathrm{a}}$ & $69,03 \pm 0,87^{\mathrm{d}}$ \\
70ST:3OHIA & $6,79 \pm 0,17^{\mathrm{c}}$ & $1,33 \pm 0,02^{\mathrm{b}}$ & $1,14 \pm 0,25^{\mathrm{b}}$ & $3,04 \pm 0,13^{\mathrm{a}}$ & $13,69 \pm 0,09^{\mathrm{c}}$ & $72,24 \pm 0,23^{\mathrm{b}}$ \\
80ST:2OHAC & $8,85 \pm 0,41^{\mathrm{a}}$ & $1,26 \pm 0,04^{\mathrm{b}}$ & $1,09 \pm 0,19^{\mathrm{b}}$ & $2,06 \pm 0,13^{\mathrm{b}}$ & $14,21 \pm 0,06^{\mathrm{b}}$ & $70,53 \pm 0,74^{\mathrm{c}}$ \\
80ST:2OHIA & $7,59 \pm 0,21^{\mathrm{b}}$ & $1,20 \pm 0,02^{\mathrm{c}}$ & $0,77 \pm 0,09^{\mathrm{c}}$ & $2,01 \pm 0,69^{\mathrm{b}}$ & $13,54 \pm 0,15^{\mathrm{d}}$ & $71,91 \pm 0,92^{\mathrm{b}}$ \\
\hline
\end{tabular}

a-d Promedios con diferente letra en la misma columna son significativamente diferentes $(p<0,05)$.

HAC: harina de amaranto crudo; HIA: harina integral de amaranto; ST: sémola de trigo;

ELN: extracto libre de nitrógeno.

En las cenizas se puede observar que hubo un incremento en comparación del control con diferencia significativa entre 70ST:30HAC, 70ST:30HIA y 80ST:20HAC. El análisis de minerales se realizó en relación con los tallarines 100\% ST, 80ST:20HIA y 70ST:30HAC. Los tallarines elaborados con harina de amaranto presentaron un incremento en minerales de $50 \%$. El contenido de hierro se in-

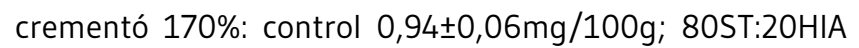
$2,37 \pm 0,59 \mathrm{mg} / 100 \mathrm{~g} ; 70 \mathrm{ST}: 30 \mathrm{HAC} 2,54 \pm 0,42 \mathrm{mg} / 100 \mathrm{~g}$. Para el calcio se incrementó $142 \%$, pues el control presentó valor de 16,90 $\pm 1,64 \mathrm{mg} / 100 \mathrm{~g} ; 80 \mathrm{ST}: 20 \mathrm{HIA} 38,93 \pm 1,03 \mathrm{mg} / 100 \mathrm{~g}$; $70 \mathrm{ST}: 30 \mathrm{HAC} 40,93 \pm 2,34 \mathrm{mg} / 100 \mathrm{~g}$. No hubo diferencia significativa $(p<0,05)$ entre el aporte de minerales de dos concentraciones de amaranto. Cuando fue comparado con el control, las formulaciones presentaron diferencia significativa.

Para la actividad de agua se observó que no hubo diferencias significativas entre bloques, pero sí entre tratamientos. El control, 80ST:20HIA y 70ST:30HIA obtuvieron una actividad de 0,344 y entre estos tres tratamientos no existió diferencia significativa. Mientras que los tratamientos $80 \mathrm{ST}: 20 \mathrm{HAC}$ y 70ST:30HIA obtuvieron un valor de 0,339 y 0,335 . El coeficiente de variación fue de $0.511 \%$ con un $\mathrm{R}^{2}$ de $86 \%$.

Los análisis microbiológicos realizados para los cinco tratamientos cumplieron con los requisitos establecidos por Ministerio de la Salud de México - MINSA ${ }^{16}$, siendo el límite máximo de $1000 \mathrm{UFC} / \mathrm{g}$ para mesófilos aerobios y $100 \mathrm{UFC} / \mathrm{g}$ para coliformes totales. Todas las muestras presentaron $<1 \log 10$ UFC/g de coliformes totales, valores menores al máximo legal permitido, cumpliendo con los parámetros de calidad sanitaria e inocuidad alimentaria. Al respecto de los mesófilos aerobios, los tratamientos se encontraron debajo del rango que es $3,7 \log 10 \mathrm{UFC} / \mathrm{g}$. El conteo de mesófilos aerobios entre los tallarines permaneció entre $2,63 \pm 0,91$ y $2,99 \pm 0,08 \log U F C / g$ sin significancia estadística $(p<0,05)$.

El comportamiento de la pasta durante y después de la cocción es un parámetro de calidad importante para los consumidores donde se observa el tiempo de cocción, aumento de peso y aumento de volumen (Tabla 2). Los valores encontrados para el aumento de peso en las muestras fueron desde 51\% (70ST:30HIA) hasta 66\% (control). La formulación 70ST:30HIA fue la única en que la ganancia de peso fue estadísticamente diferente a comparación del control. Para el aumento de volumen, el control mostró un aumento en volumen de $47 \%$, el resto de los tratamientos obtuvieron un aumento de volumen inferior, mostrando diferencia significativa entre ellos. Respecto al tiempo de cocción -que es definido como el tiempo necesario para desaparecer el color blanco en el eje central de la pasta (parte no hidratada) ${ }^{13}$ en los tratamientos, hubo diferencia tanto entre harinas agregadas como también entre tratamientos. Se observó que los tallarines 70ST:30HAC tardaron más tiempo en cocerse (20min). Seguido por el tratamiento 80ST:20HAC con $15,67 \mathrm{~min}$, el control obtuvo un valor de $12,33 \mathrm{~min}$ en llegar a 
Tabla 2. Análisis de aumento de peso (A.P.), aumento de volumen (A.V.), tiempo de cocción (T.C.), acidez alcohólica (A.A.) y análisis de color $\left(L^{*}, a^{*}, b^{*}\right)$ de los tallarines.

\begin{tabular}{l|c|c|c|c|c|c|c} 
Tratamientos & $\%$ A.P. & $\%$ A.V. & T.C. (min) & $\%$ A.A. & $L^{*}$ & $a^{*}$ & $b^{*}$ \\
\hline Control (ST) & $66,08 \pm 2,60^{\mathrm{a}}$ & $47,25 \pm 0,27^{\mathrm{a}}$ & $13,33 \pm 1,51^{\mathrm{c}}$ & $4,13 \pm 0,14^{\mathrm{c}}$ & $7209 \pm 2,03^{\mathrm{a}}$ & $1,10 \pm 0,82^{\mathrm{a}}$ & $16,22 \pm 0,19^{\mathrm{c}}$ \\
80ST:20HAC & $63,40 \pm 1,60^{\mathrm{a}}$ & $46,30 \pm 0,42^{\mathrm{b}}$ & $15,67 \pm 0,57^{\mathrm{b}}$ & $5,38 \pm 0,14^{\mathrm{a}}$ & $63,95 \pm 1,40^{\mathrm{b}}$ & $2,45 \pm 0,49^{\mathrm{c}}$ & $17,54 \pm 0,24^{\mathrm{a}}$ \\
70ST:30HAC & $63,43 \pm 2,54^{\mathrm{a}}$ & $45,36 \pm 0,13^{\mathrm{c}}$ & $20,00 \pm 1,00^{\mathrm{a}}$ & $5,42 \pm 0,13^{\mathrm{a}}$ & $64,98 \pm 2,78^{\mathrm{b}}$ & $3,17 \pm 0,81^{\mathrm{b}}$ & $17,12 \pm 0,42^{\mathrm{b}}$ \\
80ST:20HIA & $62,52 \pm 2,09^{\mathrm{a}}$ & $44,28 \pm 0,39^{\mathrm{d}}$ & $10,33 \pm 1,53^{\mathrm{dc}}$ & $4,54 \pm 0,19^{\mathrm{b}}$ & $60,81 \pm 2,53^{\mathrm{c}}$ & $4,16 \pm 0,51^{\mathrm{a}}$ & $17,63 \pm 0,67^{\mathrm{a}}$ \\
70ST:30HIA & $51,20 \pm 3,36^{\mathrm{b}}$ & $39,45 \pm 0,53^{\mathrm{e}}$ & $8,07 \pm 1,43^{\mathrm{d}}$ & $4,08 \pm 0,13^{\mathrm{c}}$ & $59,56 \pm 0,27^{\mathrm{c}}$ & $4,72 \pm 0,21^{\mathrm{a}}$ & $17,22 \pm 0,37^{\mathrm{ad}^{\mathrm{d}}}$
\end{tabular}

a-e Promedios con diferente letra en la misma columna son significativamente diferente $(p<0,05)$.

HAC: harina de amaranto crudo; HIA: harina integral de amaranto; ST: sémola de trigo.

cocción y por último los tallarines $80 \mathrm{ST}: 20 \mathrm{HIA}$ con 10,33min y 70ST:30HIA con $8,07 \mathrm{~min}$. Los resultados de acidez alcohólica mostraron que los tallarines 70ST:30HIA obtuvieron $4,08 \%$ seguido por el control con $4,13 \%$, ambos no tuvieron diferencia significativa $(p>0,05)$. Los que tuvieron mayor vaIor en la acidez alcohólica fueron los tallarines elaborados con HAC. Las proporciones 20 y $30 \%$ de HAC no tuvieron diferencia significativa ( $p>0,05)$, lo que nos indica que hubo un excelente control en los estándares de inocuidad durante la operación de procesamiento. Con respecto a los análisis de textura hubo diferencias entre las concentraciones y las harinas de amaranto utilizadas (Figura 1).

Respecto al análisis sensorial, hubo diferencia significativa entre tratamientos $(p<0,05)$ en los atributos de apariencia, textura, aroma, dulzura, sabor y aceptación general (Tabla 3). Los tratamientos se ubicaron entre 7 y 4 de la escala hedónica. Relacionado con los parámetros sensoriales, el índice de luminosidad de las pastas sustituidas fue significativamente menor para el control $\left(L^{*}=72,09\right)$, sin embargo a

Figura 1. Propiedades de textura obtenidas en el análisis de tensión (Resistencia Máxima).

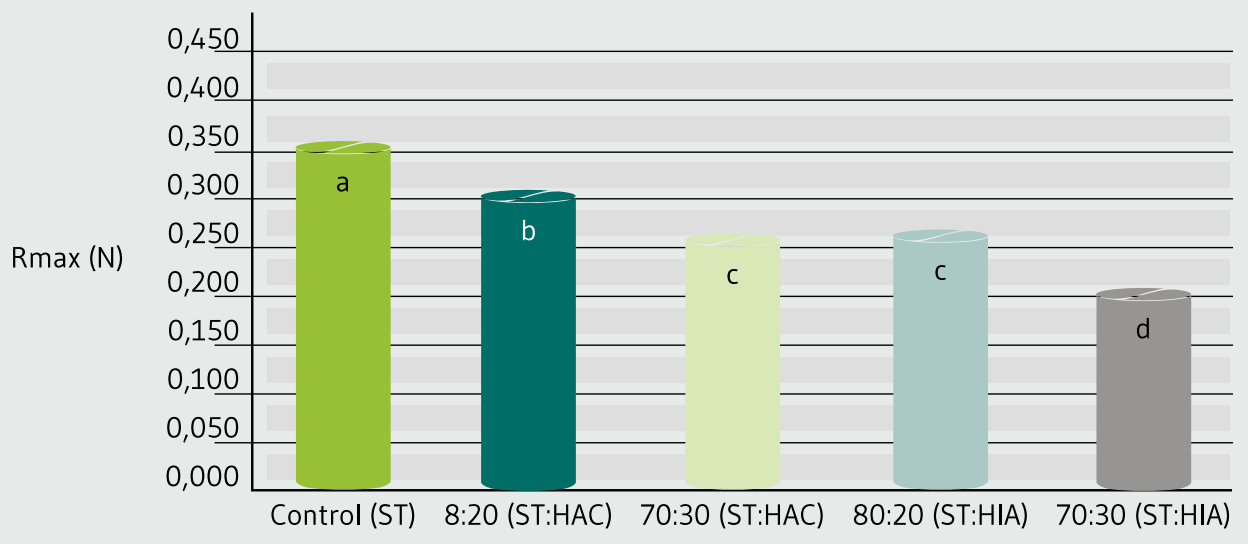

a-d: Promedios con diferente letra son significativamente diferentes $(p<0,05)$.

ST: sémola de trigo; HIA: harina integral de amaranto; HAC: harina de amaranto crudo; Rmax(N): Resistencia Máxima en Newton. 
medida que se incrementaron los niveles de sustitución de HAC y HIA también se incrementó ligeramente el color amarillo ( $b^{*}$ que varió entre los tratamientos de 16,22 para el control hasta 17,63 para el tratamiento 80ST:20HIA (Tabla 2). Con el parámetro $a^{*}$, el tratamiento 70ST:30HIA presentó mayor color rojo $(4,72)$ y el control presentó menor color rojo $(1,10)$.

Cuando se correlacionó con el análisis sensorial, se observó que a mayor incremento de luminosidad $\left(L^{*}\right)$, agradó más a los panelistas. Mientras que en $a^{*}$ y $b^{*}$ es al contrario. Mientras más rojo y amarillo tienen los tallarines, menos fueron aceptados por los panelistas.

\section{DISCUSIÓN}

Los resultados obtenidos en los análisis microbiológicos se deben a la correcta implementación de las buenas prácticas de manufactura. Esto indica que los ingredientes se mantuvieron en condiciones ambientales adecuadas para la elaboración de la pasta.

En lo que respecta la producción y composición fisicoquímica de los tallarines, es importante hacer algunos comentarios sobre proteínas, fibras, ceniza y actividad de agua. En la fabricación de pastas al igual que en panificación, la calidad de la proteína usada es más importante que la cantidad, es decir por su contenido y balance de aminoácidos limitantes, el trigo al igual que otros cereales es deficiente en lisina, en tanto el amaranto es valorado por su alto contenido de lisina, dando una mejor calidad a los tallarines. Aunque se incrementó en contenido de proteína, esto se hizo con proteínas entre cuyas características físicas no predomina la capacidad para formar matrices viscoelásticas (características propias de gluten). Acerca del contenido de fibras, se puede inferir que hubo un incremento significativo en el contenido de fibra saludable con la adición de amaranto a la formulación. El tipo de molienda hace que las harinas de amaranto tengan una diferencia entre el aporte de fibra $^{17}$. Es por eso que los tallarines elaborados con HIA tienen un menor aporte de fibra a comparación de la HAC.

Las cenizas están formadas principalmente por minerales procedentes de la parte externa del grano, que se incorporan a la harina según su tasa de extracción. El estudio de Drago y Cols. ${ }^{18}$ reporta un incremento en calcio de $180 \%$ y en hierro de $230 \%$, sustituyendo $20 \%$ de ST por HIA. El escaso aporte potencial de hierro en los tallarines se atribuye a la degradación del promotor (amasado, almacenamiento y cocción) ${ }^{18}$. Sobre la actividad de agua, valores debajo de 0,5 en pastas hacen que no haya proliferación microbiana ${ }^{19}$.

En la textura se observó que el control obtuvo mayor resistencia. El grado de absorción de agua del control fue mayor con respecto a otras harinas, de esta forma la harina integral de amaranto pudo haber dificultado la gelatinización del almidón que provocó una menor resistencia a diferencia del control y los tallarines elaborados con HAC. Al momento de la cocción de los tallarines, el almidón continúa volviéndose más espeso, más viscoso y resistente al flujo a medida que se gelatiniza. Adicionalmente, el control presentó mayor extensibilidad.

El comportamiento de la pasta durante y después de la cocción es uno de los parámetros de gran importancia para los consumidores. Los parámetros para el aumento de peso y de volumen se relacionan con la capacidad de

Tabla 3. Análisis sensorial de tallarines con diferentes atributos.

\begin{tabular}{l|c|c|c|c|c|c} 
Tratamientos & Apariencia & Textura & Aroma & Dulzura & Sabor & Aceptación \\
\hline Control (ST) & $6,90 \pm 1,58^{\mathrm{a}}$ & $6,62 \pm 1,76^{\mathrm{a}}$ & $6,77 \pm 1,56^{\mathrm{a}}$ & $7,01 \pm 1,66^{\mathrm{a}}$ & $6,81 \pm 1,66^{\mathrm{a}}$ & $6,94 \pm 1,56^{\mathrm{a}}$ \\
80ST:20HAC & $6,33 \pm 1,59^{\mathrm{b}}$ & $6,41 \pm 1,5^{\mathrm{b}}$ & $6,67 \pm 1,27^{\mathrm{a}}$ & $6,82 \pm 1,31^{\mathrm{a}}$ & $6,81 \pm 1,38^{\mathrm{a}}$ & $6,72 \pm 1,19^{\mathrm{b}}$ \\
70ST:30HAC & $6,22 \pm 1,65^{\mathrm{b}}$ & $6,41 \pm 1,59^{\mathrm{b}}$ & $6,44 \pm 1,22^{\mathrm{b}}$ & $6,27 \pm 1,33^{\mathrm{b}}$ & $6,17 \pm 1,74^{\mathrm{b}}$ & $6,36 \pm 1,29^{\mathrm{b}}$ \\
80ST:20HIA & $5,47 \pm 1,5^{\mathrm{c}}$ & $5,99 \pm 1,64^{\mathrm{b}}$ & $6,09 \pm 1,54^{\mathrm{b}}$ & $5,81 \pm 1,41^{\mathrm{c}}$ & $6,06 \pm 1,54^{\mathrm{b}}$ & $6,10 \pm 1,44^{\mathrm{c}}$ \\
70ST:30HIA & $4,01 \pm 1,77^{\mathrm{d}}$ & $4,89 \pm 2,00^{\mathrm{c}}$ & $5,32 \pm 1,72^{\mathrm{c}}$ & $4,32 \pm 1,76^{\mathrm{d}}$ & $4,85 \pm 1,87^{\mathrm{c}}$ & $4,72 \pm 1,68^{\mathrm{d}}$ \\
\hline
\end{tabular}

a-d Promedios con diferente letra en la misma columna son significativamente diferente $(p<0,05)$.

HAC: harina de amaranto crudo; HIA: harina integral de amaranto; ST: sémola de trigo. 
absorción de las masas, en la cual puede reflejar el rendimiento de la pasta. El aumento de volumen es dependiente del contenido y calidad de proteína ${ }^{20}$. El tiempo de cocción de una pasta fresca de ST debe estar entre 10 y $13 \mathrm{~min}$ para pastas artesanales ${ }^{21}$. Si están precocinadas, ocurre una disminución de tiempo de cocción en las masas. En este trabajo, el tiempo de cocción fue directamente proporcional a la cantidad de harina de amaranto crudo añadida.

Un punto importante en el análisis sensorial fue que el tratamiento menos valorizado fue el 80ST:20HI. Todos Ios tratamientos, a excepción del 70ST:30HIA, estuvieron en una escala hedónica de "me agrada poco". Esta muestra obtuvo un valor de 4,72 (me desagrada poco). Los tallarines 70ST:30HIA tuvieron disminución del valor en apariencia y textura. Esto era de esperar, dado que al disminuir la ST, se reduce el contenido de gluten y por lo tanto la consistencia, la cual determina en gran medida la pasta. Esto coincide con lo reportado por Granito y Cols. ${ }^{22}$, que detectaron que a un $50 \%$ de sustitución de ST, el producto se desintegra durante la cocción. Además de eso, en la apariencia, el control fue el mejor con 6,9 y el peor fue el tratamiento 70ST:30HIA con 4,0. Esto puede ocurrir debido a que los tallarines sustituidos con harina integral de amaranto llegaban a deshacerse y perder firmeza por la falta de gluten. Los tallarines con sustitución de harina de amaranto crudo llegaron a tener mejor apariencia en comparación con los tallarines con harina integral de amaranto y esto puede ser por el efecto que realizó el tostado. Los defectos sensoriales fueron minimizados mediante la adición de goma guar y aceite vegetal, que ayuda a mejorar la apariencia y textura. Con respecto al aroma y dulzura, el control fue el más aceptado. Todos los tratamientos, menos el tratamiento 70ST:30HIA, se encontraban entre "me agrada poco" y "me agrada moderadamente" de la escala hedónica, aunque la HIA es más dulce y tiene mejor aroma por el tostado. El sabor entre los tratamientos se encontraba entre "me agrada poco", excepto el tratamiento con 70ST:30HIA que fue evaluado con "me desagrada poco". Esto se debe a que los tallarines no fueron acompañados con otro producto, como salsas o aderezos.

El análisis de correlación entre las variables apariencia, textura, aroma, dulzura, sabor y aceptación general demostró que todos los atributos se correlacionaron de una manera alta positiva $(R>0,7)$, lo cual indica que la apariencia se relaciona con la textura, la textura con el aroma, el aroma con la dulzura, la dulzura con el sabor y la aceptación general con todos los atributos; pero el atributo sabor es el que tiene una mayor correlación.
También hubo una correlación entre apariencia del atributo del análisis sensorial con los análisis de color. El mayor incremento de luminosidad $\left(L^{*}\right)$ les agrado más a los panelistas, mientras que en $a^{*}$ y $b^{*}$ es al contrario, cuanto más rojo y amarillo tienen los tallarines menos aceptados fueron por los panelistas. Los resultados de este estudio exponen que a pesar de que los atributos de apariencia, textura, aroma, dulzura y sabor tienen correlación con la aceptación general, el atributo sabor es el que tiene una mayor correlación. La pasta al no tener un sabor específico, llega a complementarse con todo tipo de alimentos.

Además, el incremento en el color rojo en las pastas sustituidas se incrementó a medida que aumentó la sustitución. Hipotéticamente, las tonalidades de rojo se deben a que la HIA Ilevó un proceso de tostado, provocando que la reacción de Maillard actuara en los tallarines.

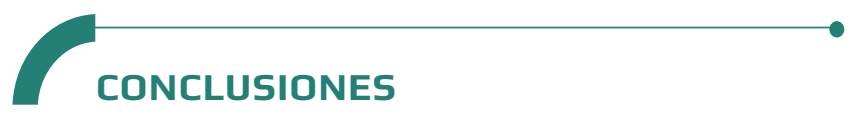

La formulación 70ST:30HIA de tallarines mostró no ser apta desde el punto de vista sensorial. Los tallarines elaborados con una sustitución hasta $20 \%$ y $30 \%$ de harina de amaranto crudo presentaron características fisicoquímicas requeridas para este producto. La sustitución parcial de $20 \%$ de harina de amaranto mejoró significativamente el contenido nutricional en fibra con un incremento de $60 \%$ y en minerales en $140 \%$. Los tallarines elaborados con una sustitución hasta $30 \%$ de harina de amaranto crudo presentaron características de calidad requerida para este producto, donde el sabor fue el factor que más influyó en la aceptación general.

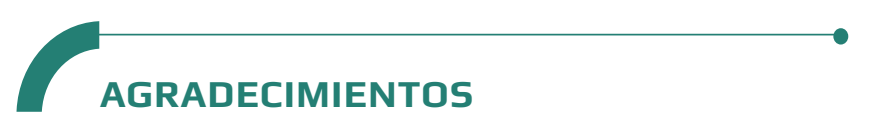

Los autores del presente estudio agradecen al Laboratorio de Análisis de Alimentos de la Escuela Panamericana de Zamorano (LAAZ) por el apoyo técnico en la ejecución y desarrollo del presente documento.

\section{CONFLICTO DE INTERESES}

Los autores expresan que no hay conflictos de interés al redactar el manuscrito. 


\section{REFERENCIAS}

(1) Sabanis D, Makri E, Doxastakis G. Effect of durum flour enrichment with chickpea flour on the characteristics of dough and lasagne. J Sci Food Agric. 2006; 86(12): 1938-44.

(2) Parra M. Canasta Familiar [Internet]. Inflacion. 2010 [citado 19 de febrero de 2016]. Disponible en: http://inflacion.com.co/ canasta-familiar.html

(3) Desrosier NW. Elementos de tecnología de alimentos. Mexico: Cecsa; 1989.

(4) Ramos Diaz JM, Suuronen J-P, Deegan KC, Serimaa R, Tuorila $H$, Jouppila K. Physical and sensory characteristics of cornbased extruded snacks containing amaranth, quinoa and kañiwa flour. Lebenson Wiss Technol. 2015; 64(2): 1047-56.

(5) Oszvald M, Tamás C, Rakszegi M, Tömösközi S, Békés F, Tamás L. Effects of incorporated amaranth albumins on the functional properties of wheat dough. J Sci Food Agric. 2009; 89(5): 8829.

(6) Alvarez-Jubete L, Auty M, Arendt EK, Gallagher E. Baking properties and microstructure of pseudocereal flours in gluten-free bread formulations. Eur Food Res Technol. 2009; 230(3): 437.

(7) Fiorda FA, Soares Jr. MS, da Silva FA, Grosmann MVE, Souto LRF. Microestructure, texture and colour of gluten-free pasta made with amaranth flour, cassava starch and cassava bagasse. Lebenson Wiss Technol. 2013; 54(1): 132-8.

(8) Kill RC, Turnbull K. Tecnología de la elaboración de pasta y sémola. Zaragoza, Spain: Acribia; 2004.

(9) Prabhasankar P, Rajiv J, Indrani D, Rao GV. Influence of whey protein concentrate, additives, their combinations on the quality and microstructure of vermicelli made from Indian $\mathrm{T}$. Durum wheat variety. J Food Eng. 2007; 80(4): 1239-45.

(10) Ross AS. Instrumental Measurement of Physical Properties of Cooked Asian Wheat Flour Noodles. Cereal Chem. 2006; 83(1): 42-51.

(11) Cunniff $P$, editor. Official methods of analysis of $A O A C$ international. 16 ed. Washington, DC: Association of Official Analytical Chemists; 1995.
(12) Feng P, Weagant SD, Grant MA, Burkhardt W. Chapter 4 Enumeration of Escherichia coli and the Coliform Bacteria. En: Bacteriological Analytical Manual. 8 ed. Silver Spring, MD, USA: U.S. Food and Drug Administration; 1998.

(13) American Association of Cereal Chemists. Approved methods of the American Association of Cereal Chemists. St. Paul, MN, USA: AACC; 1995.

(14) Agência Nacional de Vigilância Sanitária do Brasil. Resolução RDC no 12 [Internet]. Disponible en: http://www.anvisa.gov.br/ alimentos/legis/especifica/html

(15) Smewing J. Analyzing the texture of pasta for quality control. Cereal Food World. 1997; 42(1): 8-12.

(16) Ministerio de Salud de México. Permiso sanitario de funcionamiento para establecimientos y regulaciones de Máximo y Mínimo permitido Coliformes totales y Aerobios Mesófilos. 2003.

(17) Porr M. Amaranto: planta latinoamericana con fuerzas colosales. Revista Mexicana de semillas. 2012; 11-3.

(18) Wolfgor R, Drago SR, Rodriguez V, Pellegrino NR, Valencia ME. In vitro measurement of available iron in fortified foods. Food Res Int. 2002; 35(1): 85-90.

(19) Premier Analytical Services. Water Activity (aw) in Food - A Key Intrinsic [Internet]. Paslabs. 2012. Disponible en: http://www. paslabs.co.uk/info-sheets/water-activity-aw-in-food-a-keyintrinsic.html

(20) Ormenese R de CSC, de Faria EV, Gomes CR, Yotsuyanagi K. Massas Alimentícias Não-convencionais à Base de Arroz - Perfil Sensorial e Aceitação pelo Consumidor. Braz J Food Technol. 2001; 4: 67-74.

(21) Cruz RS, Soares N de FF. Efeito da adição de CO2 nas características tecnológica e sensorial do macarrão massa fresca tipo talharim. Ciênc agrotec. 2004; 28(4): 848-55.

(22) Granito M, Torres A, Guerra M. Desarrollo y evaluación de una pasta a base de trigo, maíz, yuca y frijol. INCI. 2003; 28(7): 372-9. 